\title{
Investigating the role of rare heterozygous TREM2 variants in Alzheimer's disease and frontotemporal dementia
}

\author{
Elise Cuyvers $^{\text {a,b }}$, Karolien Bettens ${ }^{\text {a,b }}$, Stéphanie Philtjens ${ }^{\text {a,b }}$, Tim Van Langenhove ${ }^{\text {a,b,c }}$, Ilse Gijselinck ${ }^{\text {a,b }}$, \\ Julie van der Zee ${ }^{\mathrm{a}, \mathrm{b}}$, Sebastiaan Engelborghs ${ }^{\mathrm{b}, \mathrm{d}}$, Mathieu Vandenbulcke ${ }^{\mathrm{e}}$, Jasper Van Dongen ${ }^{\mathrm{a}, \mathrm{b}}$, \\ Nathalie Geerts $^{\mathrm{a}, \mathrm{b}}$, Githa Maes ${ }^{\mathrm{a}, \mathrm{b}}$, Maria Mattheijssens ${ }^{\mathrm{a}, \mathrm{b}}$, Karin Peeters ${ }^{\mathrm{a}, \mathrm{b}}$, Patrick Cras $^{\mathrm{b}, \mathrm{c}}$, \\ Rik Vandenberghe $^{\mathrm{f}, \mathrm{g}}$, Peter P. De Deyn ${ }^{\mathrm{b}, \mathrm{d}, \mathrm{h}}$, Christine Van Broeckhoven ${ }^{\mathrm{a}, \mathrm{b}}$, Marc Cruts ${ }^{\mathrm{a}, \mathrm{b}}$, \\ Kristel Sleegers ${ }^{a, b}, *$, on behalf of the BELNEU consortium
}

a Neurodegenerative Brain Diseases group, Department of Molecular Genetics, VIB, Antwerp, Belgium
b Institute Born-Bunge, University of Antwerp, Antwerp, Belgium
${ }^{\mathrm{c}}$ Department of Neurology, Antwerp University Hospital, Edegem, Belgium
${ }^{\mathrm{d}}$ Department of Neurology and Memory Clinic, Hospital Network Antwerp, Middelheim and Hoge Beuken, Antwerp, Belgium
${ }^{\mathrm{e}}$ Department of Psychiatry and Memory Clinic, University Hospitals Leuven, Leuven, Belgium
${ }^{\mathrm{f}}$ Department of Neurology and Memory Clinic, University Hospitals Leuven, Leuven, Belgium
${ }_{\mathrm{g}}^{\mathrm{g}}$ Laboratory for Cognitive Neurology, Department of Neurology, University of Leuven, Leuven, Belgium
${ }^{\mathrm{h}}$ Department of Neurology and Alzheimer Research Center, University Medical Center Groningen, Groningen, the Netherlands

\section{A R T I C L E I N F O}

\section{Article history:}

Received 8 May 2013

Received in revised form 2 August 2013

Accepted 6 September 2013

\section{Keywords:}

TREM2

Alzheimer's disease

Frontotemporal dementia

Rare variants

Meta-analysis

IgV-set domain

\begin{abstract}
A B S T R A C T
Homozygous mutations in exon 2 of TREM2, a gene involved in Nasu-Hakola disease, can cause frontotemporal dementia (FTD). Moreover, a rare TREM2 exon 2 variant (p.R47H) was reported to increase the risk of Alzheimer's disease (AD) with an odds ratio as strong as that for $A P O E \varepsilon 4$. We systematically screened the TREM2 coding region within a Belgian study on neurodegenerative brain diseases (1216 AD patients, 357 FTD patients, and 1094 controls). We observed an enrichment of rare variants across TREM2 in both AD and FTD patients compared to controls, most notably in the extracellular IgV-set domain (relative risk $=3.84$ [95\% confidence interval $=1.29-11.44$ ]; $p=0.009$ for $\mathrm{AD}$; relative risk $=6.19[95 \%$ confidence interval $=1.86-20.61] ; p=0.0007$ for FTD). None of the rare variants individually reached significant association, but the frequency of p.R47H was increased $\sim 3$-fold in both AD and FTD patients compared to controls, in line with previous reports. Meta-analysis including 11 previously screened $A D$ cohorts confirmed the association of p.R47H with $\mathrm{AD}\left(p=2.93 \times 10^{-17}\right)$. Our data corroborate and extend previous findings to include an increased frequency of rare heterozygous TREM2 variations in AD and FTD, and show that TREM2 variants may play a role in neurodegenerative diseases in general.
\end{abstract}

(c) 2013 Elsevier Inc. All rights reserved.

\section{Introduction}

Current high-throughput sequencing technologies such as whole-exome and whole-genome sequencing enable the identification of rare genetic causes of disease. Using a whole-exome

The BELNEU consortium includes the following individuals: Jan De Bleecker, Patrick Santens, Anne Sieben, Bart Dermaut (University Hospital Ghent, Ghent, Belgium); Adrian Ivanoiu (Saint-Luc University Hospital, Brussels, Belgium); Olivier Deryck, Bruno Brugmans (AZ Sint-Jan Brugge, Bruges, Belgium); Jan Versijpt, Alex Michotte (University Hospital Brussels, Brussels, Belgium); Christiana Willems (Jessa Hospital, Hasselt, Belgium); Eric Salmon (University of Liège, Liège, Belgium).

* Corresponding author at: Neurodegenerative Brain Diseases Group, VIB Department of Molecular Genetics, University of Antwerp-CDE, Universiteitsplein 1, B-2610 Antwerp, Belgium. Tel.: +32 3265 1032; fax: +32 32651112.

E-mail address: kristel.sleegers@molgen.vib-ua.be (K. Sleegers). sequencing approach, homozygous mutations in the gene encoding triggering receptor expressed on myeloid cells 2 (TREM2; 6p21.1) were identified as a cause of behavioral variant frontotemporal dementia (FTD) in 3 consanguineous Turkish families (Guerreiro et al., 2012). TREM2 is a receptor of the innate immune system, expressed on the cell membrane of myeloid cells, especially on immature dendritic cells, microglia, and osteoclasts (Colonna 2003). Homozygous loss-of-function mutations in TREM2 (e.g., p.Q33X) were previously found in patients with Nasu-Hakola disease, also known as polycystic lipomembranous osteodysplasia with sclerosing leukoencephalopathy (PLOSL) (Klunemann et al. 2005; Paloneva et al., 2002; Soragna et al., 2003), a rare recessively inherited disease that is characterized by early-onset progressive dementia and bone cysts. In addition, a homozygous deletion affecting the consensus donor splice site in intron 1 has 
been reported in a Lebanese family with early-onset dementia, clinically resembling behavioral FTD, without bone involvement (Chouery et al., 2008).

More recently, an increased frequency of rare heterozygous TREM2 variations was detected in Alzheimer's disease (AD) patients in 2 independent studies (Guerreiro et al., 2013; Jonsson et al., 2013), suggesting that heterozygous TREM2 variations are risk factors for dementia with onset later in life. In line with this, 2 heterozygous carriers of a TREM2 loss-of-function mutation in a PLOSL family were reported to have subclinical evidence of impaired visuospatial memory and a selective metabolic deficit in the basal ganglia on functional neuroimaging compared to their homozygous wild-type relatives who presented normal neuropsychological and neuroimaging findings (Montalbetti et al., 2005).

Remarkable in AD patients was the increased genetic diversity across exon 2 of the gene compared to that in control individuals. Interestingly, 3 mutations were previously found in a homozygous state in context of FTD or PLOSL (p.T66M, p.Y38C and p.Q33X) (Guerreiro et al., 2012, 2013; Soragna et al., 2003). One rare variant in particular, p.R47H (rs75932628), showed a strong association with late-onset $A D$ in 2 studies $\left(p=9.0 \times 10^{-9}\right.$ (Guerreiro et al., 2013); $p=3.42 \times 10^{-10}$ ) (Jonsson et al., 2013). The effect size of this variation was as high as APOE $\varepsilon 4$ allele, although it occurs at much lower frequency (minor allele frequency $[\mathrm{MAF}]<1 \%$ ). This association was confirmed in a Spanish (Benitez et al., 2013) and a French (Pottier et al., 2013) population including both late- and early-onset $\mathrm{AD}$ patients, focusing on TREM2 exon 2, and a similar trend was observed on imputed genotype data for p.R47H in a study population originating from the United States, United Kingdom, and Europe (Giraldo et al., 2013).

TREM2 is a membrane protein that forms a receptor-signaling complex with protein tyrosine kinase binding protein. This complex is involved in the immune response and activation of macrophages/microglia and dendritic cells, resulting in phagocytosis and elevated short-term production of reactive oxygen species (Neumann and daly, 2012; Paloneva et al., 2002). TREM2 exon 2, in which both p.R47H and several homozygous FTD mutations were observed, encodes both the signal peptide and part of the extracellular domain containing an IgV-set domain. Hypothetically, nonsynonymous variations in TREM2 may lead to a disturbed immune response with extensive inflammation or defective microglial survival or function, thus contributing to the neurodegenerative process (Guerreiro et al., 2013; Otero et al., 2009). Nevertheless, because studies on AD have mostly focused on TREM2 exon 2, the frequency and relevance of mutations outside of exon 2 remains unclear. Of note, 2 homozygous mutations outside of exon 2 have previously been detected in families segregating autosomal recessive behavioral variant FTD (Chouery et al., 2008; Giraldo et al., 2013).

In the present study, we extend previous reports by systematically investigating the entire TREM2 coding region for the contribution of rare variations to the occurrence of both AD and FTD in an extensive prospective study population of Belgian dementia patients ( $\mathrm{n}=1216$ AD patients, $\mathrm{n}=357$ FTD patients) and nonaffected individuals $(\mathrm{n}=1094)$.

\section{Methods}

\subsection{Belgian study cohort}

The AD cohort consisted of 1216 AD patients (mean age of onset [AAO] $74.2 \pm 9.0$ years, $64.4 \%$ female), the majority of which was ascertained at the memory clinic of the ZNA Middelheim, Antwerpen, Belgium (P.P.D.D. and S.E.) in the frame of a prospective study of neurodegenerative and vascular dementia in Flanders, the
Dutch-speaking region of Belgium (Engelborghs et al., 2003, 2006). Consensus diagnosis of possible and probable $\mathrm{AD}$ was given by at least 2 neurologists based on the National Institute of Neurological and Communication Disorders and Stroke-Alzheimer's Disease and Related Disorders Association (NINCDS-ADRDA) criteria (McKhann et al., 1984). Another subset of AD patients was collected at the Memory Clinic of the University Hospitals of Leuven (UHL), Leuven, Belgium (M.V., R.V.) as part of a prospective study of the molecular genetics of cognitive impairment that was initiated in October 2006 using the same clinical assessments and biosampling schemes. Each patient underwent a neuropsychological examination and structural and/or functional neuroimaging (Bettens et al., 2009). Routine genetic screening of this AD cohort previously revealed 6 PSEN1 mutations, 1 APP mutation, 3 GRN null mutations, and 5 pathogenic repeat expansions in C9orf72.

For a subset of patients $(n=331$; mean AAO $75.8 \pm 8.6$ years, $62.8 \%$ female), cerebrospinal fluid (CSF) levels of amyloid- $\beta$ peptide (A $\beta$ 1-42), total tau (T-tau), and tau phosphorylated at threonine 181 (P-tau181P) were available as part of the diagnostic workup, determined with commercially available, single parameter enzyme-linked immunosorbent assay (ELISA) kits (Innogenetics, Ghent, Belgium).

The Belgian FTD cohort consisted of 357 FTD patients (mean AAO $62.6 \pm 10.0$ years; $45 \%$ female), recruited in the framework of the Belgian Neurology (BELNEU) consortium, a multicenter collaboration of dementia expertise centers from Belgium (Flanders, Wallonia, and Brussels) (Gijselinck et al., 2011; Van Langenhove et al., 2013). Index patients were evaluated using a standard protocol including a detailed clinical and family history, neurologic examination, and neuroimaging. The diagnosis of FTD was made according to established clinical criteria of Neary (Neary et al., 1998). In $5.7 \%$ of the FTD subjects, an autopsy diagnosis confirmed clinical diagnosis of FTD. Routine genetic screening previously revealed 27 pathogenic C9orf72 repeat expansions, 18 GRN null mutations, 6 MAPT mutations, 2 VCP mutations, and 1 CHMP2B mutation.

The control cohort $(n=1094$, mean age at inclusion $65.2 \pm 13.6$ years, $56.3 \%$ female) consisted of healthy individuals, who either were recruited from partners of patients visiting the Memory Clinic of ZNA Middelheim and Hoge Beuken, Antwerp (P.P.D.D. and S.E.), and the Memory Clinic at the University Hospitals of Leuven (M.V., R.V.) and screened for neurological or psychiatric antecedents, neurological complaints, or organic disease involving the central nervous system, or were community-recruited control individuals who were included after interview concerning medical and familial history and Mini Mental State Examination (MMSE>24) (Folstein et al., 1975).

All participants and/or their legal guardian gave written informed consent for participation in clinical and genetic studies. Clinical study protocol and the informed consent forms for patient ascertainment were approved by the Ethics Committee of the respective hospitals at the cohort sampling sites in Belgium. The genetic study protocols and informed consent forms were approved by the Ethics Committees of the University of Antwerp and the University Hospital of Antwerp, Belgium.

\subsection{TREM2 resequencing}

Genomic DNA was extracted from peripheral blood lymphocytes using standard procedures. Resequencing of the full TREM2 coding DNA sequence (CDS) and untranslated regions (UTR) was performed by polymerase chain reaction (PCR)-based amplification of the genomic DNA followed by Sanger sequencing of the 5 exons and intron-exon boundaries (NM_018965.2). Primers were designed using the PCR primer design tool Primer3 (Supplementary Table 1) (http://primer3.sourceforge.net/). 
All sequences were analyzed by 2 independent researchers using Seqman (DNASTAR, Madison, WI) and NovoSNP (Weckx et al., 2005) software packages. Numbering of variations at genomic DNA level was based on the GenBank Accession Number NC_000006.11, transcript level on NM_018965.2, and protein level on the GenPept Accession Number NP_061838.1.

\subsection{In silico prediction}

The effects of rare coding TREM2 variations were predicted using PolyPhen-2 (Polymorphism Phenotyping v2; http://genetics.bwh. harvard.edu/pph2/, SIFT (Sorting Intolerant From Tolerant; http:// sift.jcvi.org/www/SIFT_enst_submit.html), and SNPs\&Go (http:// snps.uib.es/snps-and-go//snps-and-go.html). PolyPhen-2 predicts a possible impact of amino acid substitutions on the structure and function of human proteins. SIFT predicts whether an amino acid substitution affects protein function based on sequence homology and physical proportions of amino acids. SNPs\&Go predicts human disease-related mutations in functionally annotated proteins.

\subsection{Statistical analysis}

For common TREM2 variants with MAF $>1 \%$, deviations from Hardy-Weinberg equilibrium (HWE) were assessed using an exact HWE test (http://www.pharmgat.org/IIPGA2/Bioinformatics/ exacthweform), and allele frequencies were compared between AD patients or FTD patients and healthy control individuals using $\chi^{2}$ statistics or Fisher's exact test. Odds ratios (OR) (calculated relative to the common genotype) and 95\% confidence intervals (95\% CI) were calculated using a logistic regression model, using SPSS 20.0 Version for Windows (IBM SPSS Inc, Chicago, IL). A 2-sided $p$ value of 0.05 or less was considered statistically significant.

Rare variant association analyses were performed by collapsing of alleles with MAF $<1 \%$ and comparing the overall frequency of rare variant alleles between patients and controls using $\chi^{2}$ statistics. Given the putative role of the IgV-set domain, we separately performed an analysis for rare variant alleles in this domain.

Fixed effects (Mantel-Haenszel) meta-analysis of p.R47H was performed based on raw allele data of the Belgian AD cohort and 11 additional AD cohorts published until August 1, 2013 (Benitez et al., 2013; Giraldo et al., 2013; Gonzalez Murcia et al., 2013; Guerreiro et al., 2013; Jonsson et al., 2013; Pottier et al., 2013). For cohorts for which allele counts were not given, these were derived from total sample sizes of patients and controls, minor allele frequency in controls, and odds ratios. The meta-analysis was performed once including and once excluding the 2 discovery cohorts (Guerreiro et al., 2013; Jonsson et al., 2013). Mantel-Haenszel summary OR, $\mathrm{I}^{2}$, and Cochran's $Q$ test for heterogeneity were computed in R using the library epiR, version $0.9-45$.

\section{Results}

\subsection{TREM2 resequencing}

Sequencing of the TREM2 CDS and UTR in 1216 AD patients, 357 FTD patients, and 1094 healthy individuals resulted in the identification of 15 rare variants (MAF $<1 \%$ ) in exons $2,3,4$, and 5 (including 3'UTR) (Table 1). No common polymorphisms with MAF $>5 \%$ were observed. Only a few of the variants were found in exons 3,4 , and 5 . Most rare variations were found in the IgV-set domain of exon 2 (Fig. 1).

\subsubsection{TREM2 IgV-set domain}

In total in the TREM2 IgV-set domain, 8 non-synonymous variations were identified, of which 5 mutations in $\mathrm{AD}(\mathrm{n}=4)$ and/or
FTD $(\mathrm{n}=2)$ only, and 3 variants in patients and non-affected individuals (Table 1 ). All variations except $\mathrm{p} . \mathrm{R} 62 \mathrm{H}$ were rare with MAF $<1 \%$.

Of the disease-specific mutations, p.Q33X was found in a heterozygous state in 1 FTD patient. In AD patients, 2 mutations were found at TREM2 amino residue 39 (p.D39G, pD39E), and 1 new variant was detected in $1 \mathrm{AD}$ patient with an AAO of 82 years (p.G58A). Mutation p.D87N was identified in 1 AD and 2 FTD patients (Table 1). According to Polyphen-2, disease-specific variants p.G58A, p.D87N, and p.D39E were considered probably and possibly damaging (Table 3 ).

Of the 3 variations that were found in patients and non-affected individuals (p.T96K, p.R47H and p.R62H), all carriers were heterozygous except for $1 \mathrm{AD}$ patient homozygous for p.R47H (Table 1). At least 1 prediction instrument considered p.R47H and p.T96K damaging (Table 3). The AD-associated variation p.R47H (Guerreiro et al., 2013; Jonsson et al., 2013) was detected at a frequency of $0.41 \%$ and $0.42 \%$ in Belgian AD and FTD patients, and at a frequency of $0.14 \%$ in Belgian controls. The AD patient homozygous for p.R47H had an AAO of 56 years, which was lower than the AAO of heterozygous p.R47H AD carriers, with onset ages ranging between 60 and 80 years. Variation p.G17E was detected in 1 non-affected individual 46 years old. Detailed clinical and pathological description of the IgV-set domain mutation carriers can be found in the Supplementary data.

\subsubsection{TREM2 variations outside the IgV-set domain}

A synonymous (p.L133L) and a non-synonymous (p.H157Y) variation were identified in TREM2 exon 3. Both variations were present in $\mathrm{AD}(0.2 \%$ and $0.08 \%)$ and FTD (0.13\% and 0.4\%). p.H157Y has not previously been observed in control individuals, and is predicted possibly damaging by PolyPhen-2 (Table 3). Three nonsynonymous but predicted benign variations were identified in TREM2 exon 4. The variant p.S162R was present only in $1 \mathrm{AD}$ patient 84 years old. Variation p.L211P was present in the AD (0.09\%), FTD $(0.28 \%)$, and control $(0.05 \%)$ populations. Of note, this variant always co-occurred with the exon 2 variant p.T96K. Variant p.T223I was present in both the $\mathrm{AD}(0.04 \%)$ and control $(0.1 \%)$ populations. Only 1 variant was observed in exon 5 (rs2234258), occurring in only 1 FTD patient. This variant is located in the 3(UTR of transcript variant 1 (c. ${ }^{*}+73 C>$ T; NM_018965; Fig. 1), with no predicted effect on expression and minimal evidence of transcription factor binding (RegulomeDB; http://www.regulomedb.org). However, in an alternative transcript (NM_001271821; Fig. 1), this mutation encodes a premature translation termination (c.676C $>$ T; p.W221X). This variation has been observed before. In the signal peptide, 1 missense mutation (p.G17E) was observed in 1 control individual only (Table 1).

\subsection{Association analysis of TREM2 variations}

The frequency of the p.R47H allele was increased approximately 3-fold in both AD and FTD patients compared to control individuals (Table 1). Nonetheless, this did not reach nominal statistical significance for AD (Fisher's exact test, $p=0.08$ ) and FTD (Fisher's exact test, $p=0.2$ ), possibly due to low frequency of p.R47H. Metaanalysis combining data from the AD cohort of this study and 11 previously screened $\mathrm{AD}$ cohorts, confirms association of $\mathrm{p} . \mathrm{R} 47 \mathrm{H}$ with $\mathrm{AD}$ (odds ratio $[\mathrm{OR}]=2.76[95 \% \mathrm{CI}=2.10-3.28] ; p=$ $\left.2.93 \times 10^{-17}\right)$. After exclusion of the 2 discovery cohorts, the metaanalysis findings remain significant $(\mathrm{OR}=3.87[95 \% \mathrm{CI}=$ 1.91-6.99]; $p=9.03 \times 10^{-5}$ ) (Fig. 2).

No significant association with AD and FTD was observed for variant p.R62H, which had a MAF of $1.9 \%$ in $A D$ patients $(\mathrm{OR}=1.54$ 
Table

TREM2 coding and untranslated region (UTR) variants in the Belgian cohort

\begin{tabular}{|c|c|c|c|c|c|c|c|c|c|c|c|c|c|c|c|c|c|}
\hline & \multirow{2}{*}{$\begin{array}{l}\text { Genomic } \\
\text { position }\end{array}$} & \multirow{2}{*}{$\begin{array}{l}\text { Protein } \\
\text { position }\end{array}$} & \multirow[t]{2}{*}{ dbSNP137 } & \multicolumn{8}{|c|}{ Belgian population } & \multicolumn{4}{|c|}{ Guerreiro et al. } & \multicolumn{2}{|l|}{ EVS } \\
\hline & & & & $\begin{array}{l}\text { Minor } \\
\text { allele } \\
\text { count } \\
\mathrm{AD}\end{array}$ & Freq AD & $\begin{array}{l}\mathrm{OR}(95 \% \mathrm{CI}) \\
p \text { value }\end{array}$ & $\begin{array}{l}\text { Minor } \\
\text { allele } \\
\text { count } \\
\text { FTD }\end{array}$ & $\begin{array}{l}\text { Freq } \\
\text { FTD }\end{array}$ & $\begin{array}{l}\mathrm{OR}(95 \% \mathrm{CI}) \\
p \text { value }\end{array}$ & $\begin{array}{l}\text { Minor } \\
\text { allele } \\
\text { count C }\end{array}$ & Freq C & $\begin{array}{l}\text { Minor } \\
\text { allele } \\
\text { count } \\
\mathrm{AD}\end{array}$ & $\begin{array}{l}\text { Freq } \\
A D\end{array}$ & $\begin{array}{l}\text { Minor } \\
\text { allele } \\
\text { count } \\
\text { Control }\end{array}$ & $\begin{array}{l}\text { Freq } \\
\text { Control }\end{array}$ & $\begin{array}{l}\text { Minor } \\
\text { allele } \\
\text { Eur.Am. } \\
\text { count }\end{array}$ & $\begin{array}{l}\text { Freq } \\
\text { Eur.Am. }\end{array}$ \\
\hline \multirow[t]{10}{*}{ Exon 2} & g.41129342C $>\mathrm{T}$ & p.G17E & - & 0 & 0.0000 & & 0 & 0.0000 & & 1 & 0.0005 & - & - & - & - & - & - \\
\hline & g. $41129295 G>A$ & p.Q33X & rs104894002 & 0 & 0.0000 & & 1 & 0.0014 & & 0 & 0.0000 & 2 & 0.0018 & 0 & 0.0000 & 1 & 0.0001 \\
\hline & g.41129276T $>C$ & p.D39G & - & 1 & 0.0004 & & 0 & 0.0000 & & 0 & 0.0000 & - & - & - & - & - & - \\
\hline & g. $41129275 G>C$ & p.D39E & rs200392967 & 2 & 0.0008 & & 0 & 0.0000 & & 0 & 0.0000 & - & - & - & - & 1 & 0.0001 \\
\hline & g. $41129252 C>T$ & p.R47H & rs75932628 & $10^{\mathrm{a}}$ & 0.0041 & $\begin{array}{l}3.01(0.83- \\
10.94) 0.08\end{array}$ & 3 & 0.0042 & $\begin{array}{l}3.07(0.62- \\
15.23) 0.2\end{array}$ & 3 & 0.0014 & 22 & 0.010 & 5 & 0.002 & 22 & 0.0026 \\
\hline & g. $41129219 C>G$ & p.G58A & - & 1 & 0.0004 & & 0 & 0.0000 & & 0 & 0.0000 & - & - & - & - & - & - \\
\hline & g. $41129133 \mathrm{C}>\mathrm{T}$ & p.D87N & rs142232675 & 1 & 0.0004 & & 2 & 0.0028 & & 0 & 0.0000 & 6 & 0.003 & 0 & 0.000 & 12 & 0.0014 \\
\hline & g. $41129105 \mathrm{G}>\mathrm{T}$ & p.T96K & rs2234253 & 2 & 0.0008 & & 2 & 0.0028 & & 1 & 0.0005 & 4 & 0.002 & 3 & 0.001 & 10 & 0.0012 \\
\hline & g.41129207C $>T$ & p.R62H & rs143332484 & $46^{\mathrm{b}}$ & 0.0189 & $\begin{array}{l}1.54(0.96 \\
-\end{array}$ & 8 & 0.0112 & $\begin{array}{l}0.91(0.41 \\
-\end{array}$ & 27 & 0.0123 & 25 & 0.011 & 31 & 0.014 & 89 & 0.0105 \\
\hline & & & & & & 2.49) 0.08 & & & $2.00) 0.81$ & & & & & & & & \\
\hline \multirow[t]{2}{*}{ Exon 3} & g.41127613C $>A$ & p.L133L & rs144250872 & 5 & 0.0020 & & 1 & 0.0013 & & 2 & 0.001 & - & - & - & - & 15 & 0.0017 \\
\hline & g. $41127543 \mathrm{G}>\mathrm{A}$ & p.H157Y & rs2234255 & 2 & 0.0008 & & 3 & 0.004 & & 0 & 0.0000 & 1 & 0.002 & 0 & 0.0000 & 0 & 0.0000 \\
\hline \multirow[t]{3}{*}{ Exon 4} & g. $41126801 \mathrm{G}>\mathrm{C}$ & p.S162R & - & 1 & 0.0004 & & 0 & 0.0000 & & 0 & 0.0000 & - & - & - & - & 1 & 0.0001 \\
\hline & g. $41126655 \mathrm{~A}>\mathrm{G}$ & p.L211P & rs2234256 & 2 & 0.0013 & & 2 & 0.0028 & & 1 & 0.0005 & 0 & 0.0000 & 3 & 0.003 & 12 & 0.0014 \\
\hline & g.41126619G $>A$ & p.T223I & rs138355759 & 1 & 0.0004 & & 0 & 0.0000 & & 2 & 0.001 & - & - & - & - & 6 & 0.0007 \\
\hline UTR & g. $41126429 C>T$ & - & rs2234258 & 0 & 0.0000 & & 1 & 0.0014 & & 0 & 0.0000 & - & - & - & - & 2 & 0.0006 \\
\hline
\end{tabular}

Key: AD, Alzheimer's disease; C, control; Cl, confidence interval; Eur.Am., European American; EVS, Exome Variant Server; Freq, frequency; OR, odds ratio.

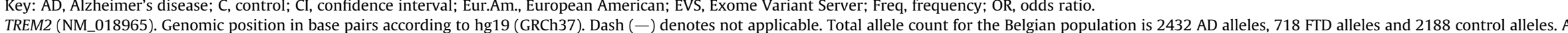

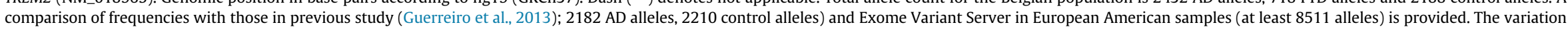
with minor allele frequency $>1 \%$ is indicated in boldface type.

a One homozygous AD patient for p.R47H.

b One homozygous AD patient for p.R62H. 
Table 2

Rare variant $(\mathrm{MAF}<1 \%)$ association analysis of TREM2

\begin{tabular}{lllllll}
\hline & $\mathrm{AD}$ & & $\mathrm{FTD}$ & \\
\cline { 2 - 3 } \cline { 5 - 6 } \cline { 5 - 6 } & $\mathrm{RR}(95 \% \mathrm{CI})$ & $p$ value & & $\mathrm{RR}(95 \% \mathrm{CI})$ & $p$ value \\
\hline TREM2 CDS + UTR & $2.54(1.23-5.23)$ & 0.009 & & $4.67(2.09-10.45)$ & 0.0000 \\
IgV-set domain & $3.84(1.29-11.44)$ & 0.009 & & $6.19(1.86-20.61)$ & 0.0007 \\
$\begin{array}{c}\text { Other TREM2 } \\
\text { variants }\end{array}$ & $1.65(0.1-4.45)$ & 0.32 & & $3.61(1.21-10.75)$ & 0.014 \\
& & & & &
\end{tabular}

Key: AD, Alzheimer's disease; CI, confidence interval; FTD, frontotemporal dementia; MAF, minor allele frequency; RR, relative risk.

Relative risks with 95\% confidence intervals are presented after collapsing alleles for rare variations (MAF $<1 \%$ ) in full TREM2 coding DNA sequence (CDS) including untranslated regions (UTR); TREM2 exon 2 encoding the IgV-set domain; and other TREM2 variants, in both the AD and FTD population compared to the control population.

[95\% CI $=0.96-2.49]$; allelic $p=0.08)$ and a MAF of $1.1 \%$ in FTD patients ( $\mathrm{OR}=0.91[95 \% \mathrm{CI}=0.41-2.0]$; allelic $p=0.81$ ).

Although none of the rare variations (MAF $<1 \%$ ) was individually associated with $\mathrm{AD}$, we observed a significant enrichment of rare variants in the CDS + UTR of TREM2 in AD patients (28 of $2432=1.15 \%$ variant alleles) compared to control individuals ( 10 of $2188=0.46 \%$ rare variant alleles) (relative risk overall $[R R]=2.54$ [95\% CI $=1.23-5.23$ ]; $p=0.009$ ) (Table 2). Analogous to AD, significantly more rare variations were found in the FTD population (15 of $718=2.1 \%$ rare variant alleles) ( $\mathrm{RR} 4.67$ [95\% CI $=$ 2.09-10.45]; $p<0.0001$ ) (Table 2). For AD patients, the increased frequency of rare TREM2 variants could be explained by an increased rare variant burden of the IgV-set domain (17 rare variant alleles, $\mathrm{RR}=3.84[95 \% \mathrm{CI}=1.29-11.44] ; p=0.009$ ) (Table 2). Three independent studies (Guerreiro et al., 2013; Jonsson et al., 2013; Pottier et al., 2013) reported sequencing data in AD/control cohorts for exon 2, which includes this domain. Meta-analysis on the total frequency of rare variants with $\mathrm{MAF}<1 \%$ in the IgV set domain resulted in a highly significant summary OR of $2.87[95 \% \mathrm{CI}=$ $2.14-3.72$ ]; $p=1.75 \times 10^{-13} ; \mathrm{I}^{2} 0 \%$, strongly influenced by $\mathrm{p} . \mathrm{R} 47 \mathrm{H}$. When excluding p.R47H carriers, this resulted in a summary OR = $2.14[95 \% \mathrm{CI}=1.00-3.49] ; p=0.05 ; \mathrm{I}^{2} 38 \%$, suggesting a residual association due to rare variants other than p.R47H in this domain of TREM2.

For the FTD patients, not only the frequency of IgV-set domain rare variants was significantly higher than for control individuals, but also the frequency of rare variants in the CDS and UTR excluding the IgV-set domain ( $\mathrm{RR}=3.61[95 \% \mathrm{CI}=1.21-10.75] ; p=0.014)$.

\section{Discussion}

Homozygous mutations in TREM2, a gene involved in a rare recessive syndrome involving very early onset dementia and bone cysts, have recently been reported to cause FTD (Guerreiro et al., 2012), and a rare TREM2 variant (p.R47H; rs75932628) was reported to increase risk of $A D$ with an effect as strong as $A P O E \varepsilon 4$ (Guerreiro et al., 2013; Jonsson et al., 2013). In this study, we investigated the contribution of variants in TREM2 in a large Belgian study on neurodegenerative brain diseases totaling 1216 Alzheimer patients, 357 FTD patients, and 1094 healthy individuals.

We found significantly more rare heterozygous TREM2 variations in both AD and FTD patients compared to control individuals. We identified 15 rare variations in TREM2 in the Belgian AD and FTD

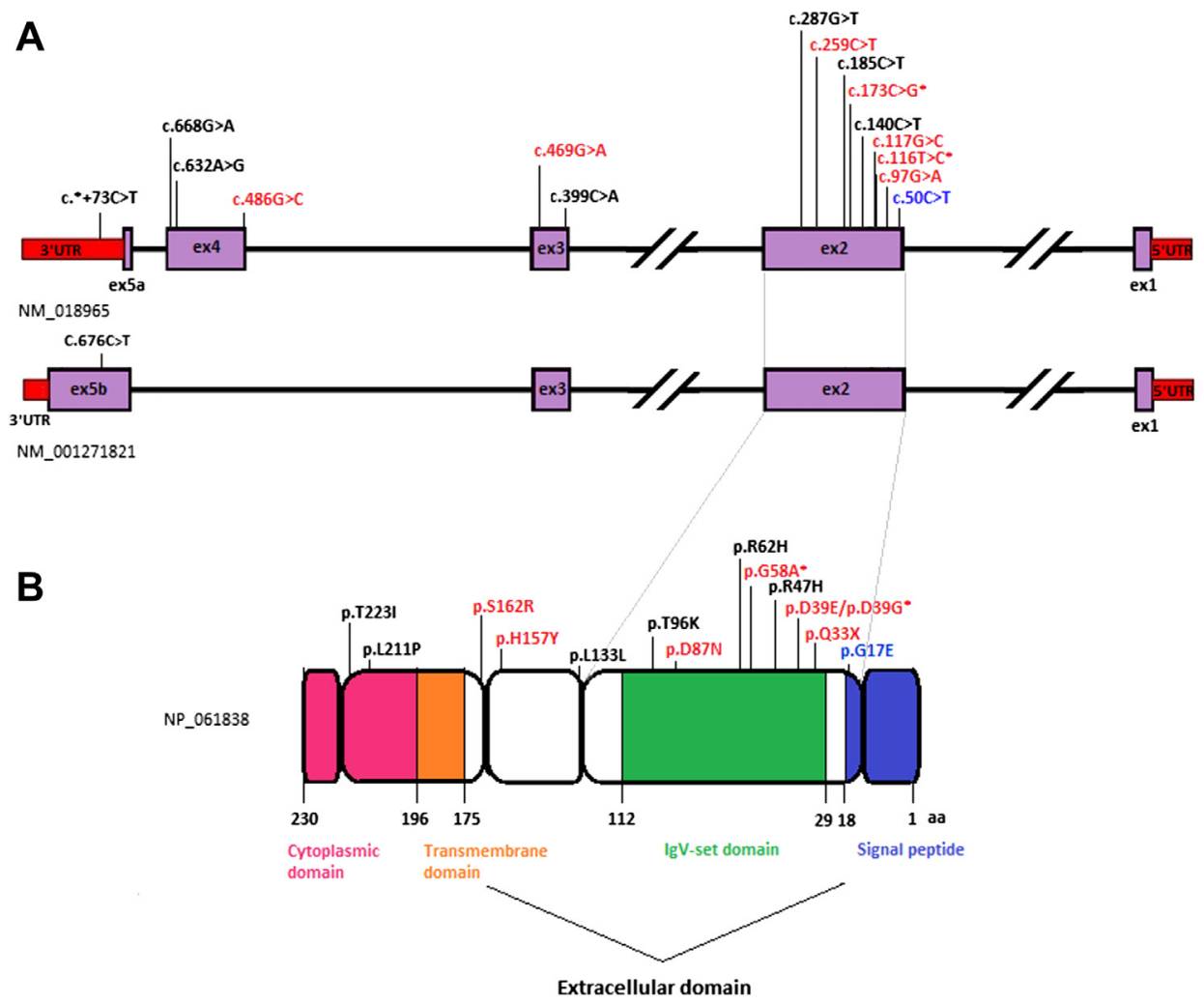

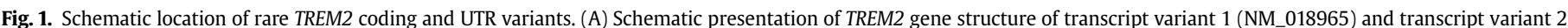

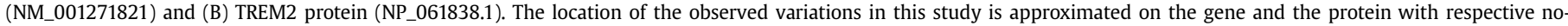

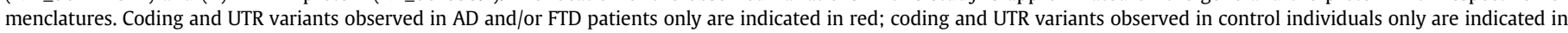

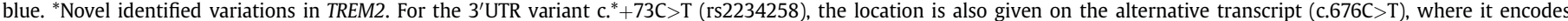

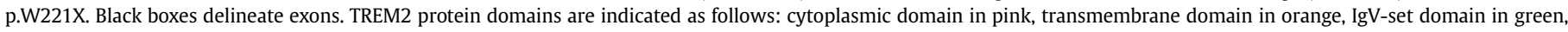
and signal peptide in blue. aa, amino acid. 
Table 3

In silico predictions of TREM2 CDS missense variants in the Belgian cohort

\begin{tabular}{|c|c|c|c|c|c|c|c|c|c|}
\hline & Exon position & Genomic position & $\begin{array}{l}\text { Protein } \\
\text { position }\end{array}$ & dbSNP137 & Polyphen-2 & SIFT & $\begin{array}{l}\text { SNPs\&Go } \\
\text { Prediction }\end{array}$ & $\begin{array}{l}\text { Reliability } \\
\text { index }\end{array}$ & Probability \\
\hline \multirow[t]{8}{*}{ Exon 2} & $\mathrm{Ex} 2+9 \mathrm{C}>\mathrm{T}$ & g.41129342C $>\mathrm{T}$ & p.G17E & - & Benign (0.166) & Tolerated (1) & Neutral & 5 & 0.226 \\
\hline & $\mathrm{Ex} 2+76 \mathrm{~T}>\mathrm{C}$ & g.41129276T $>C$ & p.D39G & - & Benign $(0.243)$ & Tolerated $(0.07)$ & Neutral & 8 & 0.100 \\
\hline & $\mathrm{Ex} 2+77 \mathrm{G}>\mathrm{C}$ & g. $41129275 G>C$ & p.D39E & rs200392967 & Possible damaging (0.892) & Tolerated $(0.4)$ & Neutral & 7 & 0.153 \\
\hline & $\mathrm{Ex} 2+100 \mathrm{C}>\mathrm{T}$ & g. $41129252 \mathrm{C}>\mathrm{T}$ & p.R47H & rs75932628 & Probably damaging (1.0) & Tolerated $(0.11)$ & Neutral & 6 & 0.187 \\
\hline & $\mathrm{Ex} 2+133 \mathrm{C}>\mathrm{G}$ & g. $41129219 C>G$ & p.G58A & - & Probably damaging (0.96) & Tolerated $(0.46)$ & Neutral & 7 & 0.154 \\
\hline & $\mathrm{Ex} 2+219 \mathrm{C}>\mathrm{T}$ & g. $41129133 \mathrm{C}>\mathrm{T}$ & p.D87N & rs142232675 & Probably damaging (1.0) & Tolerated (0.59) & Neutral & 9 & 0.054 \\
\hline & $\mathrm{Ex} 2+247 \mathrm{G}>\mathrm{T}$ & g. $41129105 \mathrm{G}>\mathrm{T}$ & p.T96K & rs2234253 & Probably damaging (1.0) & Damaging $(0)$ & Neutral & 6 & 0.220 \\
\hline & Ex2 $+145 C>T$ & g.41129207C $>T$ & p.R62H & rs143332484 & Benign (0.02) & Tolerated $(0.65)$ & Neutral & 9 & 0.043 \\
\hline Exon 3 & $\mathrm{Ex} 3+78 \mathrm{G}>\mathrm{A}$ & g. $41127543 \mathrm{G}>\mathrm{A}$ & p.H157Y & rs2234255 & Possible damaging (0.734) & Tolerated $(0.11)$ & Neutral & 8 & 0.114 \\
\hline \multirow[t]{3}{*}{ Exon 4} & $\mathrm{ex} 4+4 \mathrm{G}>\mathrm{C}$ & g. $41126801 G>C$ & p.S162R & - & Benign (0.421) & Tolerated $(0.34)$ & Neutral & 8 & 0.076 \\
\hline & ex4+150 A $>G$ & g. $41126655 \mathrm{~A}>\mathrm{G}$ & p.L211P & rs2234256 & Benign (0.001) & Tolerated (0.3) & Neutral & 10 & 0.016 \\
\hline & $\mathrm{ex} 4+186 \mathrm{G}>\mathrm{A}$ & g.41126619G $>A$ & p.T223I & rs138355759 & Benign (0.005) & Tolerated (0.52) & Neutral & 9 & 0.028 \\
\hline
\end{tabular}

Key: SNP, single nucleotide polymorphism.

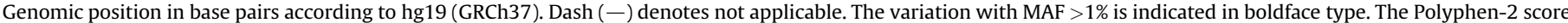

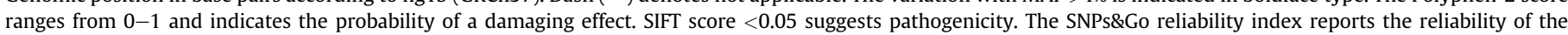
prediction, scoring from 0 (unreliable) to 10 (reliable). If disease probability is $>0.5$, the variation is predicted as disease associated.

study population, of which 2 were novel patient-specific variations (p.D39G and p.G58A). Only 1 variation had a MAF $>1 \%$ (p.R62H). We did not find a statistically significant association of p.R47H with AD, but the OR for the risk allele was in line with previous reports on TREM2 p.R47H. Furthermore, the meta-analysis, which shows a notable absence of heterogeneity, argues in favor of a role for p.R47H in AD risk even after exclusion of the 2 discovery cohorts (Guerreiro et al., 2013; Jonsson et al., 2013). We therefore conclude that the absence of association in our AD cohort is most likely due to the low frequency of the risk allele in the Belgian population, affecting statistical power to detect association. The OR for TREM2 p. $\mathrm{R} 47 \mathrm{H}(\mathrm{OR}=3.01)$ in our $\mathrm{AD}$ cohort is comparable to APOE \&4 $(\mathrm{OR}=3.3)$, in line with what was previously stated (Jonsson et al., 2013); however, because of its low risk allele frequency, the impact of TREM2 p.R47H at the population level is much lower than for $A P O E \& 4$. In our AD study population for example, the estimated population-attributable fraction (PAF) of TREM2 p.R47H is only $0.55 \%$, compared with an estimated PAF $>30 \%$ for APOE $\varepsilon 4$.

Interestingly, we observed a similarly increased frequency of p.R47H in the FTD patients as in AD patients, suggesting that this variation may play a role in neurodegenerative dementia in general.
An independent study (Rayaprolu et al., 2013) recently reported a statistically significant association between TREM2 p.R47H and FTD, underscoring our observation. Pooling both studies results in a summary OR of $4.27(95 \% \mathrm{CI}=1.64-10.73 ; p=0.003$; data not shown).

The increased overall frequency of rare TREM2 variants in the AD cohort was driven by a significantly increased burden of variants in the IgV-set domain of exon 2, underscoring the observation of Guerreiro et al. (Guerreiro et al., 2013). Remarkably, in the Belgian FTD cohort, we also observed an increased frequency of rare heterozygous variants, which was more pronounced than in the Belgian $\mathrm{AD}$ cohort, indicating that TREM2 may be a general risk factor for neurodegenerative processes underlying both $\mathrm{AD}$ and FTD. In contrast to the AD cohort, we observed significant enrichment of rare variants throughout the full CDS and UTR in the FTD cohort. Our data suggest that not only homozygous but also rare heterozygous TREM2 variants contribute to the risk of developing FTD. Because of the relatively small size of our FTD cohort to detect association with rare variants, additional studies are needed to confirm this association. To our knowledge, no studies have reported a systematic screening of the TREM2 coding region, or exon

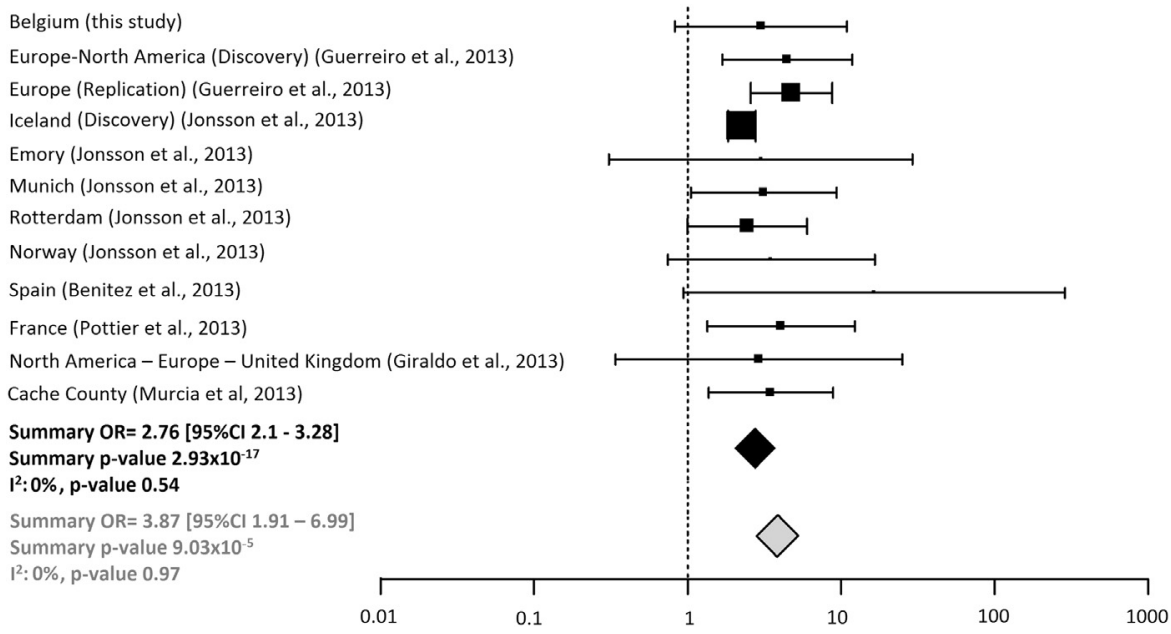

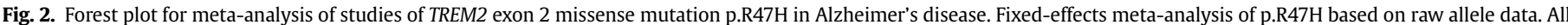

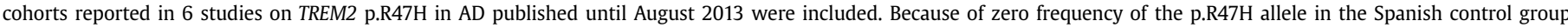

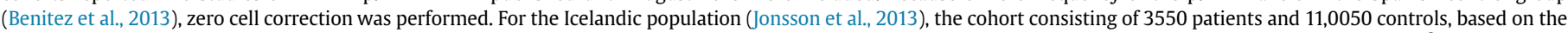

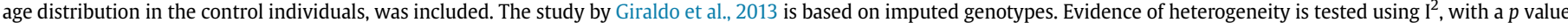

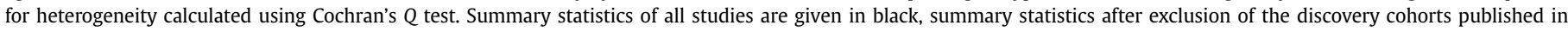
Guerreiro et al., 2013 and Jonsson et al., 2013 are shown in gray. 
Table 4

Patient characteristics of TREM2 IgV-set domain variation carriers

\begin{tabular}{|c|c|c|c|c|c|c|}
\hline Variation & ID & Diagnosis & Onset age (y) & Family history & APOE & Mutations in other dementia genes \\
\hline p.Q33X & DR806.1 & FTD (unspecified) & 64 & Positive & 44 & \\
\hline \multirow[t]{2}{*}{ p.D39E } & DR800.1 & $\mathrm{AD}$ & 73 & $\mathrm{U}$ & 33 & \\
\hline & DR801.1 & $A D$ & 52 & Positive & 23 & \\
\hline p.D39G & DR802.1 & $A D$ & 66 & Positive & 24 & \\
\hline p.G58A & DR803.1 & $\mathrm{AD}$ & 82 & $\mathrm{U}$ & 44 & \\
\hline \multirow[t]{3}{*}{ p.D87N } & DR804.1 & $\mathrm{AD}$ & $\mathrm{U}$ & $\mathrm{U}$ & 33 & \\
\hline & DR40.1 & FTD & 44 & Positive & 33 & VCP p.R159H \\
\hline & DR807.1 & FTD (unspecified) & 62 & $\mathrm{U}$ & 34 & \\
\hline p.R47H & 12 patients & $\begin{array}{l}\operatorname{AD}(\mathrm{n}=9) \\
\operatorname{FTD}(\mathrm{n}=3)\end{array}$ & $56-80^{a}$ & Positive in $20 \%$ & $\varepsilon 4: 33 \%{ }^{\mathrm{b}}$ & C9orf72 expansion (1 FTD) \\
\hline \multirow[t]{4}{*}{ p.T96K } & DR455.1 & $\mathrm{AD}$ & 76 & Negative & 34 & \\
\hline & DR805.1 & $\mathrm{AD}$ & 88 & Negative & 34 & \\
\hline & DR491.1 & FTD & 62 & Negative & 34 & \\
\hline & DR737.1 & FTD & 68 & Positive & $\mathrm{U}$ & C9orf72 expansion \\
\hline p.R62H & 53 Patients & $\begin{array}{l}\operatorname{AD}(\mathrm{n}=45) \\
\operatorname{FTD}(\mathrm{n}=8)\end{array}$ & $54-85^{a}$ & Positive in $21 \%$ & $\varepsilon 4: 38 \%{ }^{\mathrm{b}}$ & $\begin{array}{l}\text { C9orf72 expansions ( } 1 \mathrm{AD}, 1 \mathrm{FTD}) \text {; } \\
\text { PSEN1 p.A79V ( } 1 \mathrm{AD})\end{array}$ \\
\hline
\end{tabular}

Key: AD, Alzheimer's disease; APOE, apolipoprotein E; FTD, frontotemporal dementia; U, unknown.

a Range of onset ages is given for AD and FTD patients.

b $\mathrm{APOE} \varepsilon 4$ genotype frequency in both AD and FTD patients.

2, for heterozygous variants in FTD patients and ethnically matched control individuals, precluding a replication of our results. One study (Lattante et al., 2013) screened a smaller sample of 175 FTD patients, which was enriched for early AAO and recessive mode of inheritance. Two homozygous rare variants in TREM2 were reported in an FTD patient of Algerian descent. No ethnically matched control samples were tested.

Of note, none of the patient-specific mutations observed in our cohort were homozygous, including the nonsense mutation p.Q33X, which was previously identified to cause recessive PLOSL (Soragna et al., 2003) or FTD (Guerreiro et al., 2012). This mutation predicts the synthesis of a C-truncated protein, lacking the major part of the protein (197 amino acids) including the transmembrane and cytoplasmic domains, leading to loss of function of TREM2. Perhaps the partial loss-of-function of TREM2 due to a heterozygous mutation contributes to a less severe phenotype. In patients homozygous for P.Q33X, onset of dementia occurred in the second to third decade of life, whereas the Belgian FTD patient heterozygous for p.Q33X had an AAO of 63 years. Of interest, 2 heterozygous carriers of p.Q33X in an Italian PLOSL family, aged 20 and 43 years, showed a subclinical selective deficit in visuospatial memory and a mild hypoperfusion of the right basal ganglia on SPECT (Montalbetti et al., 2005). We detected another variant in an FTD patient that gives rise to a nonsense variant p.W221X, but only in an alternative transcript of TREM2 (NM_001271821; Fig. 1), which lacks the transmembrane domain, possibly encoding a soluble form of TREM2. Located in the alternative exon 5 of TREM2, this variant is not predicted to give rise to nonsense-mediated decay, but probably leads to a C-truncated form of TREM2, lacking the last 9 aminoacids. Soluble TREM2 has been observed to be increased in cerebrospinal fluid in neuroinflammatory conditions and has been suggested to counteract membrane-bound TREM2 receptor activity, attenuating the inflammatory response (Piccio et al., 2008).

Although several AD and FTD patients carrying TREM2 variants had a positive family history for dementia, DNA of relatives for segregation analysis was only available for 1 FTD patient carrying p.D87N (DR40.1; Table 4). This patient carried both TREM2 p.D87N and a previously published pathogenic VCP mutation p.R159H (van der Zee et al., 2009). This VCP mutation causes the syndrome of inclusion body myopathy, Paget's disease, and frontotemporal dementia (IBMPFD). Two siblings available for segregation analysis (1 presenting with FTD with Paget's, the other with Paget's disease only) carried VCP p.R159H but not TREM2 p.D87N (Supplemental Figure). The patient carrying both VCP p.R159H and TREM2
p.D87N had a clinical phenotype of FTD only, in contrast to both siblings not carrying TREM2 p.D87N, but had a comparable early onset age. Brain immunohistochemistry had been performed previously for this patient and the sibling with both FTD and Paget's disease. No remarkable differences were noted (van der Zee et al., 2009). Interpretation of the absence of segregation data in this pedigree is not straightforward given the presence of another syndrome involving dementia. However, the association data suggest that rare heterozygous TREM2 variants act as moderately penetrant risk factors rather than highly penetrant pathogenic mutations, which is compatible with lack of segregation.

In 1 other FTD patient carrying a heterozygous TREM2 variation another causal variation was found, a pathological $\mathrm{G}_{4} \mathrm{C}_{2}$ expansion in C9orf72 (Gijselinck et al., 2011). Both VCP and C9orf72 mutations are known to cause mixed clinical phenotypes including amyotrophic lateral sclerosis and IBMPFD, but the 2 TREM2 variant carriers had a clinical phenotype of pure behavioral FTD. Also in $2 \mathrm{AD}$ patients, carrying TREM2 p.R62H, a second pathological mutation was present, that is, a pathological C9orf72 expansion (Cacace et al., 2013) and PSEN1 p.A79V. Perhaps heterozygous TREM2 variants act as a modifier of the phenotype caused by a second (pathogenic) mutation, for example, resulting in a predominant dementia phenotype; however, to address this hypothesis, additional studies are required.

Review of the clinical and pathological records of the TREM2 rare variant carriers (Supplemental data) does not highlight signs or symptoms that appear characteristic for an underlying TREM2 mutation. The onset age of carriers varies widely, from $44-82$ years for patient-specific mutations and from 56-80 years for carriers of p.R47H. Of note, the youngest p.R47H-carrying AD patient was homozygous for this variant, which could indicate a dose effect on disease severity, but with only 1 patient this remains speculative. Numerous patients had white matter lesions on neuroimaging, which would be compatible with the observations in PLOSL (Paloneva et al., 2001) and FTD due to homozygous TREM2 mutations (Guerreiro et al., 2012), although white matter lesions are prevalent in $\mathrm{AD}$ and are as such not a likely discriminating factor. Previously described autopsies of 3 TREM2 variant carriers seemed to highlight amyloid angiopathy (Guerreiro et al., 2013), but this was not substantial in the 2 Belgian patients who came to autopsy.

In conclusion, we did not observe an association between TREM2 p.R47H and $A D$, probably due to the low occurrence of this variant in the Belgian population, but a meta-analysis in $\mathrm{AD}$ confirms the original reports. In addition, we extend these findings to include 
FTD. We report an increased frequency of rare variants across exon 2 in FTD (including a heterozygous carrier of p.Q33X), and, to a lesser extent, $\mathrm{AD}$, including 2 previously unreported mutations, suggesting that TREM2 variants may confer risk for neurodegenerative diseases in general. The pathogenic nature and mode of action of TREM2 in the neurodegenerative process remains to be elucidated, but the identification of these novel TREM2 mutations may facilitate this process.

\section{Disclosure statement}

The authors declare that they have no conflicts of interest.

\section{Acknowledgements}

Research in the authors' group is funded in part by the Interuniversity Attraction Poles program of the Belgian Science Policy Office (BELSPO, http://www.belspo.be/), the Foundation for Alzheimer Research (SAO-FRA, http://alzh.org/), the Queen Elisabeth Medical Foundation (QEMF), a Methusalem Excellence Program of the Flemish Government (EWI, http://www.ewi-vlaanderen.be/), the Research Foundation Flanders (FWO, http://www.fwo.be/), the Agency for Innovation by Science and Technology Flanders (IWT), the University Research Fund (UA, http://www.ua.ac.be/), the Medical Research Foundation Antwerp, Belgium, and the MetLife Foundation Award (C.V.B.) E.C. is a PhD fellow of IWT. K.B., I.G., and J.vdZ. are postdoctoral fellows and S.P. is a predoctoral fellow of the FWO, and R.V. is a senior clinical investigator of the FWO.

\section{Appendix A. Supplementary data}

Supplementary data associated with this article can be found, in the online version, at http://dx.doi.org/10.1016/j.neurobiolaging. 2013.09.009.

\section{References}

Benitez, B.A., Cooper, B., Pastor, P., Jin, S.C., Lorenzo, E., Cervantes, S., Cruchaga, C., 2013. TREM2 is associated with the risk of Alzheimer's disease in Spanish population. Neurobiol. Aging 34, 1711.e15-7.

Bettens, K., Brouwers, N., Van Miegroet, H., Gil, A., Engelborghs, S., De Deyn, P.P., Vandenberghe, R., Van Broeckhoven, C., Sleegers, K., 2009. Follow-up study of susceptibility loci for Alzheimer's disease and onset age identified by genomewide association. J. Alzheimers Dis. 19, 1169-1175.

Cacace, R., Van Cauwenberghe, C., Bettens, K., Gijselinck, I., van der Zee, J., Engelborghs, S., Vandenbulcke, M., Van Dongen, J., Baumer, V., Dillen, L., Mattheijssens, M., Peeters, K., Cruts, M., Vandenberghe, R., De Deyn, P.P., Van Broeckhoven, C., Sleegers, K., 2013. C9orf72 G(4)C(2) repeat expansions in Alzheimer's disease and mild cognitive impairment. Neurobiol. Aging 34, 1712.e1-7.

Chouery, E., Delague, V., Bergougnoux, A., Koussa, S., Serre, J.L., Megarbane, A., 2008. Mutations in TREM2 lead to pure early-onset dementia without bone cysts. Hum. Mutat. 29, E194-E204.

Colonna, M., 2003. TREMs in the immune system and beyond. Nat. Rev. Immunol. 3, $445-453$.

Engelborghs, S., Dermaut, B., Goeman, J., Saerens, J., Marien, P., Pickut, B.A., van den Broeck, M., Serneels, S., Cruts, M., Van Broeckhoven, C., De Deyn, P.P., 2003. Prospective Belgian study of neurodegenerative and vascular dementia: APOE genotype effects. J. Neurol. Neurosurg. Psychiatry 74, 1148-1151.

Engelborghs, S., Dermaut, B., Marien, P., Symons, A., Vloeberghs, E., Maertens, K., Somers, N., Goeman, J., Rademakers, R., van den Broeck, M., Pickut, B., Cruts, M., Van Broeckhoven, C., De Deyn, P.P., 2006. Dose dependent effect of APOE epsilon4 on behavioral symptoms in frontal lobe dementia. Neurobiol. Aging 27, $285-292$.

Folstein, M.F., Folstein, S.E., McHugh, P.R., 1975. "Mini-mental state”. A practical method for grading the cognitive state of patients for the clinician. J. Psychiatr. Res. 12, 189-198.

Gijselinck, I., Van Langenhove, T., van der Zee, J., Sleegers, K., Philtjens, S., Kleinberger, G., Janssens, J., Bettens, K., Van Cauwenberghe, C., Pereson, S., Engelborghs, S., Sieben, A., De Jonghe, P., Vandenberghe, R., Santens, P., De Bleecker, J., Maes, G., Baumer, V., Dillen, L., Joris, G., Cuijt, I., Corsmit, E., Elinck, E., Van Dongen, J., Vermeulen, S., van den Broeck, M., Vaerenberg, C., Mattheijssens, M., Peeters, K., Robberecht, W., Cras, P., Martin, J.J., De Deyn, P.P., Cruts, M., Van Broeckhoven, C., 2011. A C9orf72 promoter repeat expansion in a
Flanders-Belgian cohort with disorders of the frontotemporal lobar degeneration-amyotrophic lateral sclerosis spectrum: a gene identification study, Lancet Neurol. 11, 54-65.

Giraldo, M., Lopera, F., Siniard, A.L., Corneveaux, J.J., Schrauwen, I., Carvajal, J. Munoz, C., Ramirez-Restrepo, M., Gaiteri, C., Myers, A.J., Caselli, R.J., Kosik, K.S. Reiman, E.M., Huentelman, M.J., 2013a. Variants in triggering receptor expressed on myeloid cells 2 are associated with both behavioral variant frontotemporal lobar degeneration and Alzheimer's disease. Neurobiol. Aging 34, 2077.e11-8.

Gonzalez Murcia, J.D., Schmutz, C., Munger, C., Perkes, A., Gustin, A., Peterson, M., Ebbert, M.T., Norton, M.C. Tschanz, J.T., Munger, R.G., Corcoran, C.D., Kauwe, J.S 2013. Assessment of TREM2 rs75932628 association with Alzheimer's disease in a population-based sample: the Cache County Study. Neurobiol. Aging 34 2889.e11-3.

Guerreiro, R., Wojtas, A., Bras, J., Carrasquillo, M., Rogaeva, E., Majounie, E., Cruchaga, C., Sassi, C., Kauwe, J.S., Younkin, S., Hazrati, L., Collinge, J., Pocock, J., Lashley, T., Williams, J., Lambert, J.C., Amouyel, P., Goate, A., Rademakers, R., Morgan, K., Powell, J., St George-Hyslop, P., Singleton, A., Hardy, J., 2013. TREM2 variants in Alzheimer's disease. N. Engl. J. Med. 368, 117-127.

Guerreiro, R.J., Lohmann, E., Bras, J.M., Gibbs, J.R., Rohrer, J.D., Gurunlian, N., Dursun, B., Bilgic, B., Hanagasi, H., Gurvit, H., Emre, M., Singleton, A., Hardy, J., 2012. Using exome sequencing to reveal mutations in TREM2 presenting as a frontotemporal dementia-like syndrome without bone involvement. Arch. Neurol. 70, 78-84.

Jonsson, T., Stefansson, H., Steinberg, S., Jonsdottir, I., Jonsson, P.V., Snaedal, J., Bjornsson, S., Huttenlocher, J., Levey, A.I., Lah, J.J., Rujescu, D., Hampel, H. Giegling, I., Andreassen, O.A., Engedal, K., Ulstein, I., Djurovic, S., IbrahimVerbaas, C., Hofman, A., Ikram, M.A., van Duijn, C.M., Thorsteinsdottir, U., Kong, A., Stefansson, K., 2013. Variant of TREM2 associated with the risk of Alzheimer's disease. N. Engl. J. Med. 368, 107-116.

Klunemann, H.H., Ridha, B.H., Magy, L., Wherrett, J.R., Hemelsoet, D.M., Keen, R.W. De Bleecker, J.L., Rossor, M.N., Marienhagen, J., Klein, H.E., Peltonen, L., Paloneva, J., 2005. The genetic causes of basal ganglia calcification, dementia, and bone cysts: DAP12 and TREM2. Neurology 64, 1502-1507.

Lattante, S., Le Ber, I., Camuzat, A., Dayan, S., Godard, C., Van Bortel, I., De Septenville, A., Ciura, S., Brice, A., Kabashi, E., 2013. TREM2 mutations are rare in a French cohort of patients with frontotemporal dementia. Neurobiol. Aging 34, 2443.e1-2.

McKhann, G., Drachman, D., Folstein, M., Katzman, R., Price, D., Stadlan, E.M., 1984 Clinical diagnosis of Alzheimer's disease: Report of the NINCDS-ADRDA Work Group under the auspices of Department of Health and Human Services Task Force on Alzheimer's Disease. Neurology 34, 939-944.

Montalbetti, L., Ratti, M.T., Greco, B., Aprile, C., Moglia, A., Soragna, D., 2005. Neuropsychological tests and functional nuclear neuroimaging provide evidence of subclinical impairment in Nasu-Hakola disease heterozygotes. Funct. Neurol. 20, 71-75.

Neary, D., Snowden, J.S., Gustafson, L., Passant, U., Stuss, D., Black, S., Freedman, M. Kertesz, A., Robert, P.H., Albert, M., Boone, K., Miller, B.L., Cummings, J. Benson, D.F., 1998. Frontotemporal lobar degeneration: a consensus on clinical diagnostic criteria. Neurology. 51, 1546-1554.

Neumann, H., Daly, M.J., 2012. Variant TREM2 as risk factor for Alzheimer's disease. N. Engl. J. Med. 368, 182-184.

Otero, K., Turnbull, I.R., Poliani, P.L., Vermi, W., Cerutti, E., Aoshi, T., Tassi, I., Takai, T. Stanley, S.L., Miller, M., Shaw, A.S., Colonna, M., 2009. Macrophage colonystimulating factor induces the proliferation and survival of macrophages via a pathway involving DAP12 and beta-catenin. Nat. Immunol. 10, 734-743.

Paloneva, J., Autti, T., Raininko, R., Partanen, J., Salonen, O., Puranen, M., Hakola, P. Haltia, M., 2001. CNS manifestations of Nasu-Hakola disease: a frontal dementia with bone cysts. Neurology 56, 1552-1558.

Paloneva, J., Manninen, T., Christman, G., Hovanes, K., Mandelin, J., Adolfsson, R, Bianchin, M., Bird, T., Miranda, R., Salmaggi, A., Tranebjaerg, L., Konttinen, Y., Peltonen, L., 2002. Mutations in two genes encoding different subunits of a receptor signaling complex result in an identical disease phenotype. Am. J. Hum. Genet. 71, 656-662.

Piccio, L., Buonsanti, C., Cella, M., Tassi, I., Schmidt, R.E., Fenoglio, C., Rinker, J. Naismith, R.T., Panina-Bordignon, P., Passini, N., Galimberti, D., Scarpini, E., Colonna, M., Cross, A.H., 2008. Identification of soluble TREM-2 in the cerebrospinal fluid and its association with multiple sclerosis and CNS inflammation. Brain 131, 3081-3091.

Pottier, C., Wallon, D., Rousseau, S., Rovelet-Lecrux, A., Richard, A.C., RollinSillaire, A., Frebourg, T., Campion, D., Hannequin, D., 2013. TREM2 R47H variant as a risk factor for early-onset Alzheimer's disease. J. Alzheimers. Dis. 35, 45-49.

Rayaprolu, S., Mullen, B., Baker, M., Lynch, T., Finger, E., Seeley, W.W., Hatanpaa, K.J., Lomen-Hoerth, C., Kertesz, A., Bigio, E.H., Lippa, C., Josephs, K.A., Knopman, D.S. White III, C.L., Caselli, R., Mackenzie, I.R., Miller, B.L., Boczarska-Jedynak, M., Opala, G., Krygowska-Wajs, A., Barcikowska, M., Younkin, S.G., Petersen, R.C. Ertekin-Taner, N., Uitti, R.J., Meschia, J.F., Boylan, K.B., Boeve, B.F., GraffRadford, N.R., Wszolek, Z.K., Dickson, D.W., Rademakers, R., Ross, O.A., 2013. TREM2 in neurodegeneration: evidence for association of the p.R47H variant with frontotemporal dementia and Parkinson's disease. Mol. Neurodegener. 8, 19.

Soragna, D., Papi, L., Ratti, M.T., Sestini, R., Tupler, R., Montalbetti, L., 2003. An Italian family affected by Nasu-Hakola disease with a novel genetic mutation in the TREM2 gene. J. Neurol. Neurosurg. Psychiatry 74, 825-826.

van der Zee, J., Pirici, D., Van Langenhove, T., Engelborghs, S., Vandenberghe, R. Hoffmann, M., Pusswald, G., van den Broeck, M., Peeters, K., Mattheijssens, M., Martin, J.J., De Deyn, P.P., Cruts, M., Haubenberger, D., Kumar-Singh, S., 
Zimprich, A., Van Broeckhoven, C., 2009. Clinical heterogeneity in 3 unrelated families linked to VCP p.Arg159His. Neurology 73, 626-632.

Van Langenhove, T., van der Zee, J., Gijselinck, I., Engelborghs, S., Vandenberghe, R. Vandenbulcke, M., De Bleecker, J., Sieben, A., Versijpt, J., Ivanoiu, A., Deryck, O., Willems, C., Dillen, L., Philtjens, S., Maes, G., Baumer, V., van den Broeck, M., Mattheijssens, M., Peeters, K., Martin, J.J., Michotte, A., Santens, P., De Jonghe, P.,
Cras, P., De Deyn, P.P., Cruts, M., Van Broeckhoven, C., 2013. Distinct clinical characteristics of C9orf72 expansion carriers compared with GRN, MAPT, and nonmutation carriers in a Flanders-Belgian FTD cohort. JAMA Neurol., 1-9.

Weckx, S., Del-Favero, J., Rademakers, R., Claes, L., Cruts, M., De Jonghe, P., Van Broeckhoven, C., De Rijk, P., 2005. novoSNP, a novel computational tool for sequence variation discovery. Genome Res. 15, 436-442. 